
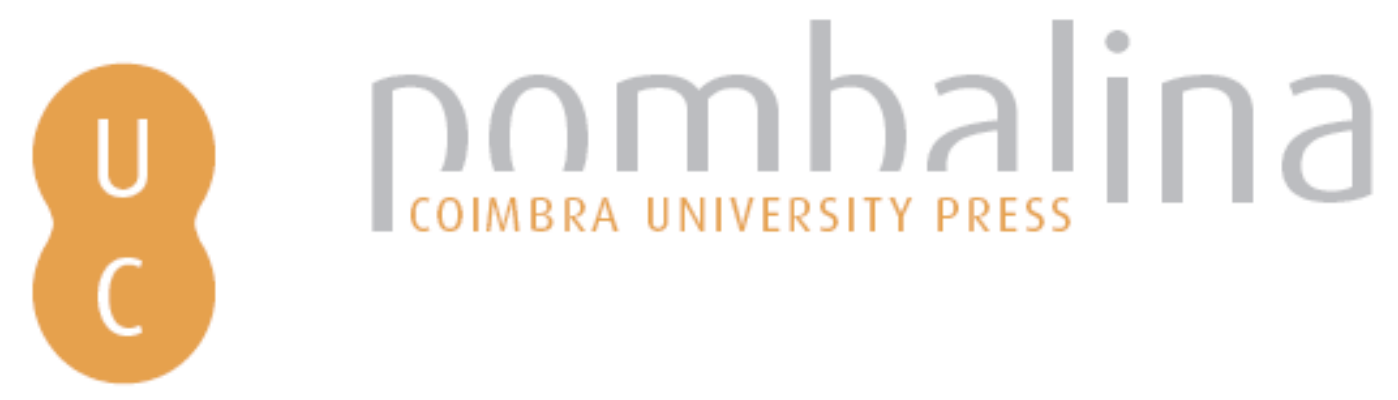

\title{
Avaliação da qualidade posicional: aplicação a um levantamento realizado em Coimbra utilizando VANT
}

Autor(es): $\quad$ Henriques, Maria João; Manta, Virgínia; Marnoto, João

Publicado por: Imprensa da Universidade de Coimbra

URL

persistente: URI:http://hdl.handle.net/10316.2/37096

DOI: $\quad$ DOI:http://dx.doi.org/10.14195/978-989-26-0983-6_43

Accessed : $\quad$ 26-Apr-2023 11:59:39

A navegação consulta e descarregamento dos títulos inseridos nas Bibliotecas Digitais UC Digitalis, UC Pombalina e UC Impactum, pressupõem a aceitação plena e sem reservas dos Termos e Condições de Uso destas Bibliotecas Digitais, disponíveis em https://digitalis.uc.pt/pt-pt/termos.

Conforme exposto nos referidos Termos e Condições de Uso, o descarregamento de títulos de acesso restrito requer uma licença válida de autorização devendo o utilizador aceder ao(s) documento(s) a partir de um endereço de IP da instituição detentora da supramencionada licença.

Ao utilizador é apenas permitido o descarregamento para uso pessoal, pelo que o emprego do(s) título(s) descarregado(s) para outro fim, designadamente comercial, carece de autorização do respetivo autor ou editor da obra.

Na medida em que todas as obras da UC Digitalis se encontram protegidas pelo Código do Direito de Autor e Direitos Conexos e demais legislação aplicável, toda a cópia, parcial ou total, deste documento, nos casos em que é legalmente admitida, deverá conter ou fazer-se acompanhar por este aviso.

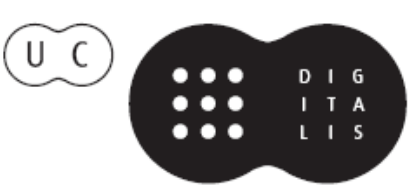




\section{$\forall$ \\ TAS DAS I JORNADAS LUSÓFONAS DE CIÊNCIAS E TECNOLOGIAS DE INFORMAÇÃO GEOGRÁFICA}

Editores

José Gomes dos Santos

Cidália Fonte

Rui Ferreira de Figueiredo

Alberto Cardoso

Gil Gonçalves

José Paulo Almeida

Sara Baptista 


\title{
ARTIGO 43
}

\section{AVAliação da QUALIdAde posicional. APlicaÇão A Um LEVANTAMENTO REALIZADO EM COIMBRA UTILIZANDO VANT}

HENRIQUES, Maria João ${ }^{1}$; MANTA, Virgínia ${ }^{2}$ E MARNOTO, João ${ }^{3}$

\author{
${ }^{1}$ Núcleo de Geodesia Aplicada - Laboratório Nacional de Engenharia Civil; Av. do Brasil 101, $1700-066$ \\ Lisboa, Portugal; Tel.: +351 218443396 email: mjoao@lnec.pt \\ ${ }^{2}$ Gabinete de Cadastro e Solos; Câmara Municipal de Coimbra; Praça 8 de Maio, 3000-300 Coimbra, Portugal \\ Tel.:+351239828078 email: virginia.manta@cm-coimbra.pt \\ 3 Novageo Solutions, S.A.; Estrada da Ponte n ${ }^{\circ}$, Quinta Grande - Alfragide; 2610-141 Amadora, Portugal \\ Tel.+351214213262 email: joao.marnoto@novageo.pt
}

\section{RESUMO}

A utilização de VANT (Veículos Aéreos Não Tripulados) como plataformas para a obtenção de fotografias destinadas à aquisição de informação geográfica tem despertado um interesse crescente por poder ser, em certos domínios, uma alternativa interessante aos métodos aéreos tradicionais. Na comunicação é apresentado o controlo da qualidade de um ortomosaico e de um modelo digital de superfície de uma zona central de Coimbra seguindo duas metodologias de análise: a habitual, baseada na análise da estatística "erro médio quadrático" e uma complexa que envolve métodos de inferência estatística.

Concluiu-se que um ortofotomapa ou uma planta, produtos com grande escala (pois é nesta área que se integram os ortomosaicos produzidos pelos VANT da gama dos utilizados em Coimbra), produzidos a partir de fotografias recolhidas por equipamento fotogramétrico de elevada qualidade, numa plataforma altamente estável e com equipamento de posicionamento muito preciso, complementados com edição rigorosa são elementos de suporte, que se considera insubstituíveis em diversas atividades de gestão do território. No entanto, para trabalhos de atualização, planeamento ou verificação, um ortomosaico e a nuvem de pontos produzidos pelos VANT são fontes de informação que, localmente, em áreas mais 
restritas, podem incluir informação mais completa e nalguns casos até mais rigorosa que a obtida a partir de métodos clássicos de produção cartográfica.

PALAVRAS-CHAVE

VANT, Ortomosaico, Modelo digital de superfície, Controlo da qualidade.

\title{
EVAluation OF THE POSITIONAL QUALITY. APPLICATION TO A SURVEY CONDUCTED IN COIMBRA USING UAV
}

\begin{abstract}
The use of UAVs (Unmanned Aerial Vehicles) as platforms for obtaining photographs aimed to cartographic production has aroused increasing interest because it can be in certain areas, an interesting alternative to traditional aerial methods. This communication presents the quality control of an orthomosaic and a digital surface model of a central zone of Coimbra, following two methods of statistical analysis: the usual, based on the "Root Mean Square Error" and another one involving methods of statistical inference.

It was concluded that large scale orthophotomaps or plans (the equipment and methodology used in the Coimbra tests originate large scale data), produced from photographs taken by photogrammetric equipment of high quality, a platform highly stable and very precise positioning equipment, complemented with rigorous editing are supporting elements, which are considered irreplaceable in various land management activities. However, for updating purposes, planning or checking, an orthomosaic and the point cloud produced by the UAV are data sources that, locally, in more restricted areas, may include more complete information and in some cases even more rigorous than what is obtained from classical methods of cartographic production.
\end{abstract}

\section{KEYWORDS}

UAV, Orthomosaic, Digital surface model, Quality control.

\section{INTRODUÇÃO}

No âmbito de uma demonstração da aplicação da tecnologia dos VANT na produção de informação geográfica, foi realizado em 2013 um voo para obter fotografias aéreas da zona da Praça da República e do Parque de Santa Cruz, em Coimbra. As fotografias aéreas foram depois alvo de um processamento que teve por objetivo a criação de um ortomosaico e do modelo digital de superfície. 
Na presente comunicação são apresentados os resultados do controlo da qualidade dos produtos gerados, para o qual foram aplicadas duas metodologias baseadas em métodos de inferência estatística: a habitual, baseada na análise da estatística "erro médio quadrático", e uma mais complexa e completa.

\section{O VEículo AÉreo NÃo TRIPUlAdo}

As fotografias do levantamento aéreo foram obtidas por uma câmara digital Canon IXUS 220 HS, montada na plataforma SenseFly Swinglet $C A M$ (Figura 1), uma aeronave muito leve $(500 \mathrm{~g})$ e pequena $(80 \mathrm{~cm}$ de envergadura). Esta aeronave pode voar de forma autónoma, desde que a trajetória de voo tenha sido previamente definida e enviada para a aeronave ou telecomandada. Durante toda a missão a aeronave emite periodicamente dados do voo, nomeadamente o nível de carga da bateria, a velocidade do vento, a atitude (obtida de uma unidade de navegação inercial), a posição (obtida a partir do recetor GPS incorporado), para uma estação de controlo. Esta consiste no software eMotion (da Sensefly), instalado num computador portátil com uma antena de rádio externa para as comunicações. Uma descrição mais completa do sistema pode ser obtida em Vallet et al. (2011).

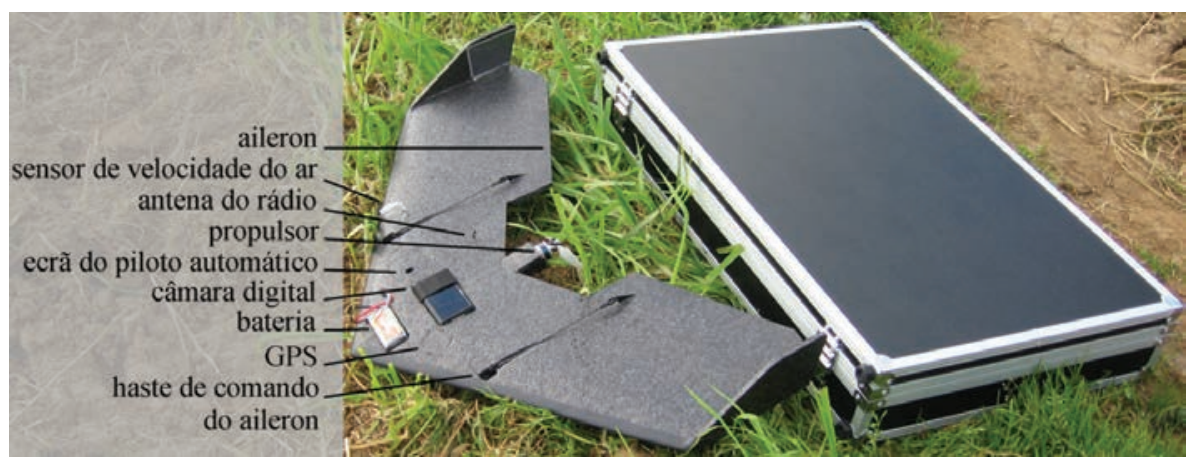

Figura 1 - SenseFly Swinglet CAM e mala de transporte 


\section{O VOO REAlizado PELO VANT SOBRE CoImbra. PROCESSAMENTO dAS}

\section{IMAGENS}

O voo foi realizado no âmbito da sessão técnica de demonstração "Veículos aéreos não tripulados (VANT) para produção de Informação Geográfica” organizada pela Secção Regional do Centro da Ordem dos Engenheiros (OERC) em janeiro de 2013. Os participantes na sessão tiveram oportunidade de seguir todos os passos, desde a programação do voo, à sua realização e ao processamento dos dados para obtenção de um ortomosaico e do modelo digital de superfície. Estes três passos são exatamente os mesmos que os seguidos em levantamentos fotogramétricos convencionais.

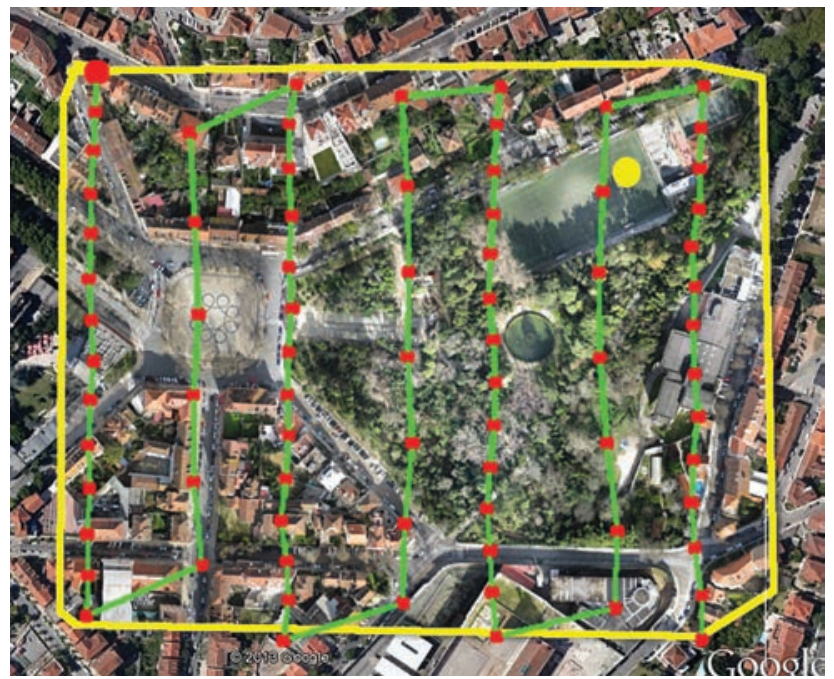

Figura 2 - Indicação da área coberta pelo ortomosaico (limite linha amarela), da zona onde a aeronave levantou e aterrou (ponto amarelo), da trajetória aproximada durante a tomada das fotografias (linha verde), dos pontos onde foram tiradas as fotografias (pontos vermelhos)

Etapas do levantamento e geração de produtos cartográficos:

- Programação do voo. O voo foi programado utilizando o software eMotion, tendo sido escolhida: i) a zona que seria coberta pelo voo, a qual incluía a Praça da República e o Parque de Santa Cruz (área aproximada 18ha); ii) a resolução pretendida (4cm por pixel); iii) a sobreposição la- 
teral e longitudinal (60\% e 90\%). Com base nestes elementos o software escolheu automaticamente a trajetória, a altura do voo e a periodicidade da obtenção das fotografias. Os dados do plano de voo foram transmitidos para a aeronave antes do voo.

- Realização do voo. A aeronave foi lançada (e aterrou) no Campo de Santa Cruz ${ }^{1}$. Na subida, e para pousar, a aeronave percorreu uma trajetória em hélice até atingir a altura de voo estabelecida (a altura é avaliada por um sensor de pressão). Uma vez esta atingida a aeronave deslocou-se até ao primeiro ponto do plano de voo, percorrendo depois a trajetória escolhida, utilizando para tal as coordenadas GPS (latitude e longitude) que estava permanentemente a calcular. Na extremidade de cada fiada, enquanto a aeronave dava a volta antes de começar nova fiada, não foram obtidas fotografias. Através da posição dos centros das fotografias apresentadas na Figura 2 verifica-se que durante o voo o vento estava predominantemente de sul e era constante.

- Após o voo, as fotografias foram descarregadas da câmara para o computador onde já se encontravam os ficheiros com dados do voo (hora, coordenadas, altura, atitude, velocidade do vento), criado com os dados emitidos pelo VANT durante o voo), e os parâmetros de calibração da câmara, calculados pela SenseFly. O processamento foi efetuado em duas etapas pelo software PostFlight Terra $3 D$ v2.2.6 (T3D) da SenseFly. Na primeira, o pré-processamento, permitiu avaliar se as condições do voo tinham sido adequadas, nomeadamente se a área tinha sido suficientemente coberta por fotografias. Na segunda fase, gerou uma nuvem de pontos, um modelo digital de superfície (MDS) e um ortomosaico. O software verificou que as 76 fotografias tiradas durante o voo eram de qualidade pois foi capaz de obter parâmetros de calibração para todas as imagens, o que tornou possível a sua integração no processamento. Determinou também a cobertura obtida: de acordo com imagem da Figura 3, quase toda a área (mais de 99\%) tinha sido coberta por três ou mais fotografias sendo que mais de $50 \%$ tinha sido coberta por cinco ou mais fotografias (Figura 4).

Principalmente devido às rajadas de vento que provocam alterações súbitas na direção do percurso e na inclinação do UAV, existem zonas

${ }^{1}$ Campo de futebol no Parque do mesmo nome 
da área levantada onde não ocorreu a sobreposição entre fotografias prevista na programação do voo enquanto noutras zonas a sobreposição foi superior ao programado.

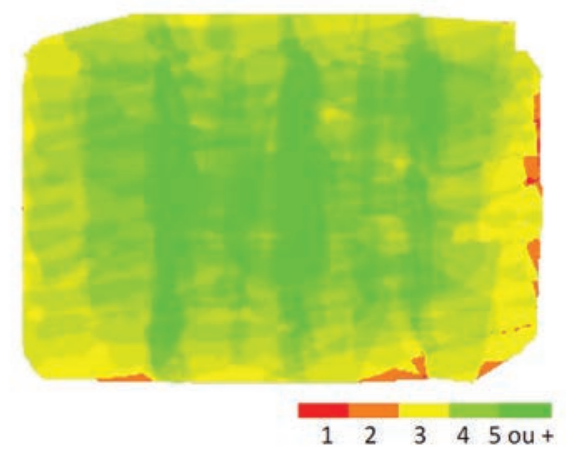

Figura 3 - Sobreposição das fotografias

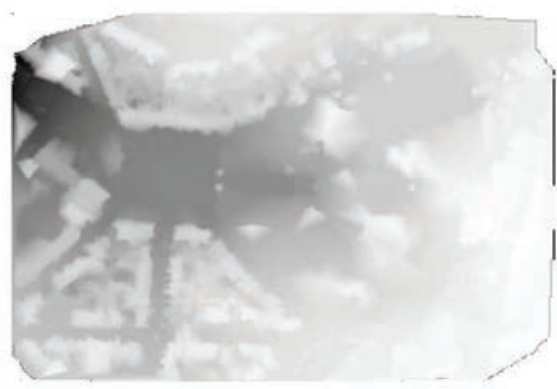

Figura 4 - MDS obtido no préprocessamento, antes da densificação

O ortomosaico representa uma projeção ortogonal da superfície do terreno. Deste é somente possível obter coordenadas planimétricas ( $\mathrm{M}$ e P). Para produzir um ortomosaico são utilizadas duas ferramentas de processamento fotogramétrico: a ortorretificação e a ligação entre fotografias (em inglês stitching). Segundo Strecha (2011) as etapas são as seguintes: i) procura automática de pontos homólogos nas imagens que foram integradas no projeto;

ii) atribuição de coordenadas, pré-determinadas, a alguns pontos (designados Pontos Fotogramétricos - PF), realizada pelo utilizador com um grande apoio do software; iii) cálculo dos parâmetros de orientação externa (estimação da posição e atitude da câmara para cada imagem) através de uma triangulação em bloco por feixes perspetivos, cálculo realizado com dados dos pontos, identificados na primeira etapa, e com informação do voo, transmitida pelo UAV (posição e atitude); iv) cálculo das coordenadas 3D de todos os pontos; v) geração do MDS; vi) criação do ortomosaico georreferenciado sendo que cada pixel é projetado no plano de acordo com o MDS. Dado que pixéis vizinhos podem ter origem em imagens diferentes o software pode aplicar uma alteração da luminosidade das 
imagens de forma a obter-se o ortomosaico com luminosidade uniforme.

Durante o processamento são calculados os parâmetros de auto-calibração (Figura 5), necessários para corrigir o efeito das distorções da lente nas imagens, os quais são apresentados no relatório produzido automaticamente pelo software. Neste relatório são também incluídos os valores iniciais, frequentemente resultantes de uma calibração realizada pela empresa que vendeu o sistema. No caso particular da distância focal o software efetua um teste simples tendo como base a diferença entre o valor inicial e o valor otimizado (o calculado), a qual não deverá ser superior a 5\% do valor inicial da distância focal.

\begin{tabular}{|c|c|c|c|c|c|c|c|c|}
\hline & $\begin{array}{l}\text { Focal } \\
\text { length }\end{array}$ & $\begin{array}{l}\text { Principal } \\
\text { point X }\end{array}$ & $\begin{array}{l}\text { Principal } \\
\text { point } Y\end{array}$ & $\mathrm{RD} 1$ & $\mathrm{RD} 2$ & RD 3 & TD 1 & TD2 \\
\hline $\begin{array}{l}\text { initial } \\
\text { values }\end{array}$ & $\begin{array}{l}2839.640[p i x] \\
4.400[\mathrm{~mm}]\end{array}$ & $\begin{array}{l}2019.760 \text { [pix] } \\
3.068 \text { [mm] }\end{array}$ & $\begin{array}{l}1547.000 \text { [pix] } \\
2.251[\mathrm{~mm}]\end{array}$ & -0.043 & 0.026 & -0.006 & 0.001 & 0.002 \\
\hline $\begin{array}{l}\text { optimized } \\
\text { values }\end{array}$ & $\begin{array}{l}2816.477[p i x] \\
4.364[\mathrm{~mm}]\end{array}$ & $\begin{array}{l}2035.681 \text { [pix] } \\
3.044[\mathrm{~mm}]\end{array}$ & $\begin{array}{l}1461.928 \text { [pix] } \\
2.383[\mathrm{~mm}]\end{array}$ & -0.042 & 0.021 & -0.002 & -0.003 & 0.003 \\
\hline
\end{tabular}

Figura 5 - Parâmetros da câmara

O ortomosaico foi georreferenciado no sistema PT-TM06 por atribuição de coordenadas a pontos bem identificados nas imagens (por exemplo, traços sobre pavimentos ou desenhos da calçada), pontos designados por pontos fotogramétricos (PF), segunda etapa descrita no anterior parágrafo. Foram utilizados nove pontos cujas coordenadas foram obtidas pelo método GPS/RTK: a estação móvel foi materializada por um recetor Topcon GR3 colocado no topo de um bastão (Figura 6); a estação base, pertencente à Câmara Municipal de Coimbra, estava situada no edifício dos Paços do Concelho, a cerca de $800 \mathrm{~m}$ de distância do local.

A identificação dos PF nas imagens foi efetuada pelo utilizador, sendo que este, para cada PF, identificava a sua localização numa foto. Seguidamente o software identificava automaticamente o ponto em todas as fotos onde este aparecia, ou deveria aparecer (Figura 7). O operador tinha oportunidade de verificar se nas fotos os pontos ficaram bem identificados podendo, se necessário, alterar para a posição mais correta. 

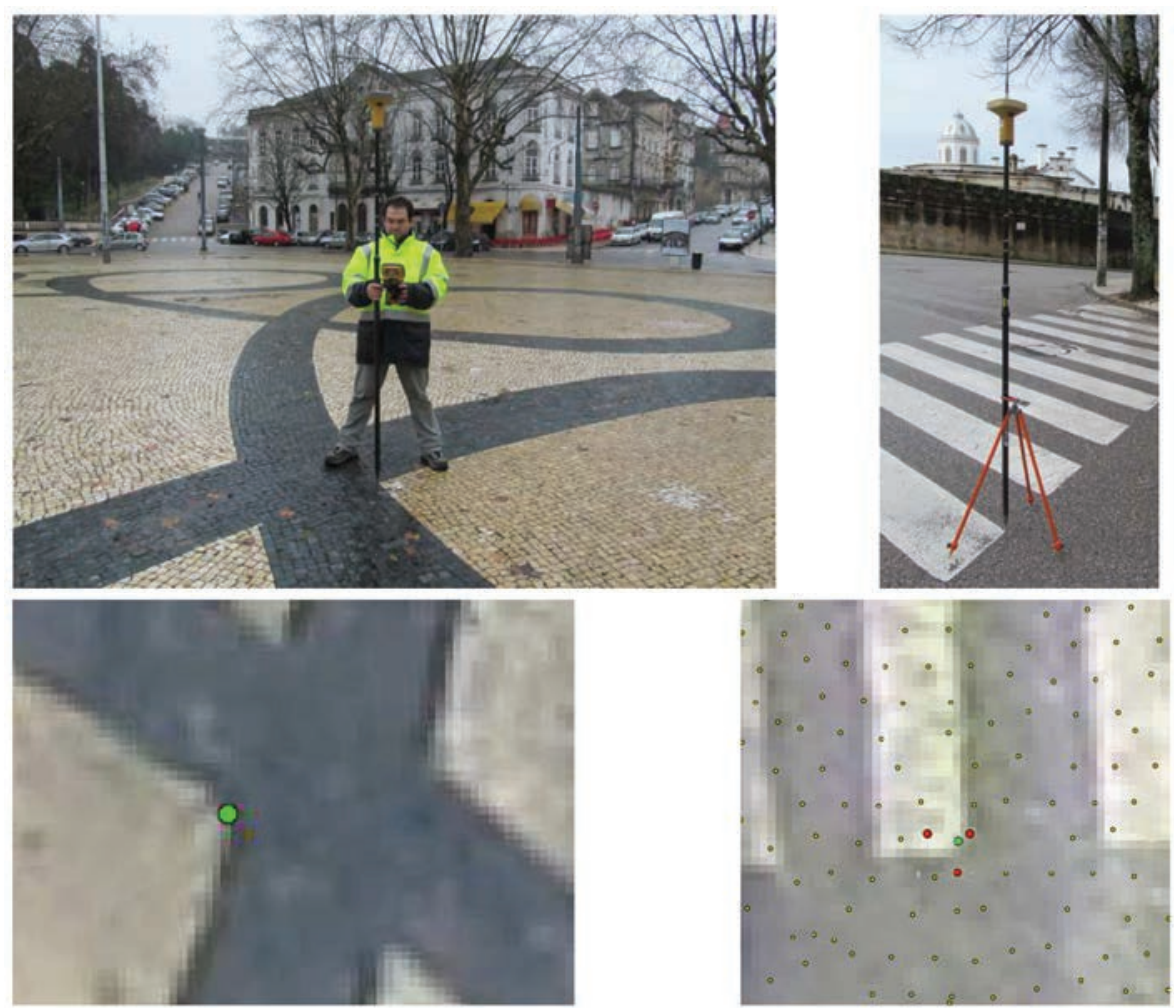

Figura 6 - Coordenação de dois pontos (na Praça de República e numa passadeira). Identificação dos pontos correspondentes nas imagens (pontos verdes) e nuvem de pontos com indicação, a vermelho, dos três pontos utilizados no cálculo da altitude baricêntrica
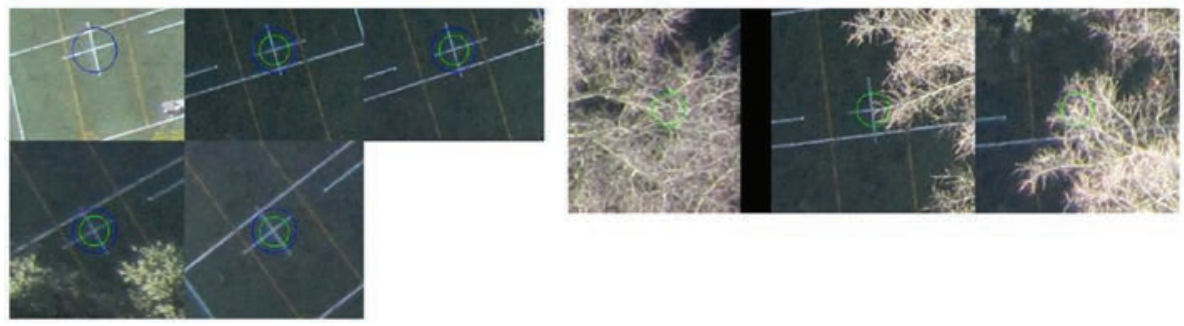

Figura 7 - Identificação de um ponto de forma automática pelo software T3D. Primeiro grupo: cinco fotos onde o software identificou o ponto; segundo grupo: três fotos onde o ponto não foi identificado por estar coberto por vegetação

Muito importante para a qualidade final dos produtos produzidos (ortomosaico e MNS) é a capacidade do software para o reconhecimento de pontos homólogos nas diferentes imagens. Para tal contribuem três 
fatores decisivos: a qualidade do algoritmo que efetua a correlação de imagens, os materiais que compõem as superfícies fotografadas e a iluminação. Também muito influente na qualidade é a densidade dos pontos na nuvem de pontos (pontos com coordenadas 3D). Se em algumas áreas é fácil verificar as razões para não existirem pontos a definir a superfície (em zonas muito uniformes ou cobertas por árvores) noutras é difícil perceber os motivos para não aparecerem pontos (caso de telhados em telha). Na Figura 8 apresenta-se o efeito do pavimento em pedra branca, envolvente ao edifício da OERC; em contrapartida, a cobertura do edifício à sua direita permitiu a geração de um número muito elevado de pontos. As zonas com asfalto preto e as com árvores e arbustos são más para localizar pontos; em oposição, nalgumas coberturas em relva pode-se encontrar uma densidade elevada de pontos. Na Figura 9 verifica-se que em dois telhados semelhantes coexistem situações opostas, respetivamente baixa e elevada densidade de pontos.
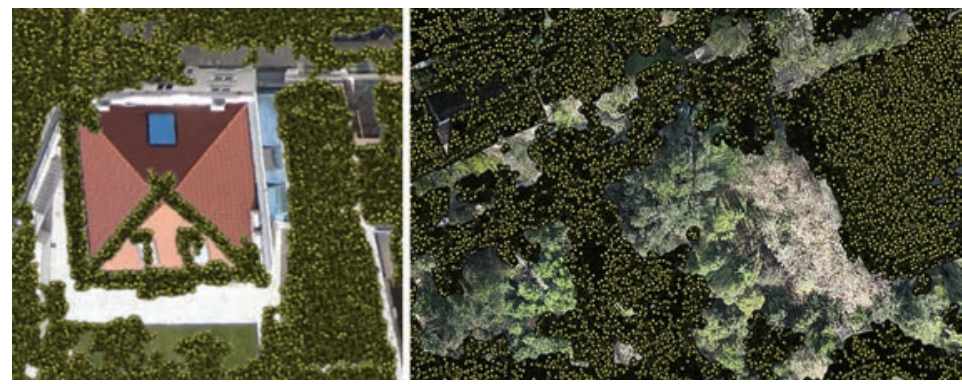

Figura 8 - Exemplos de zonas sem pontos: edifício da OERC e Parque de Santa Cruz

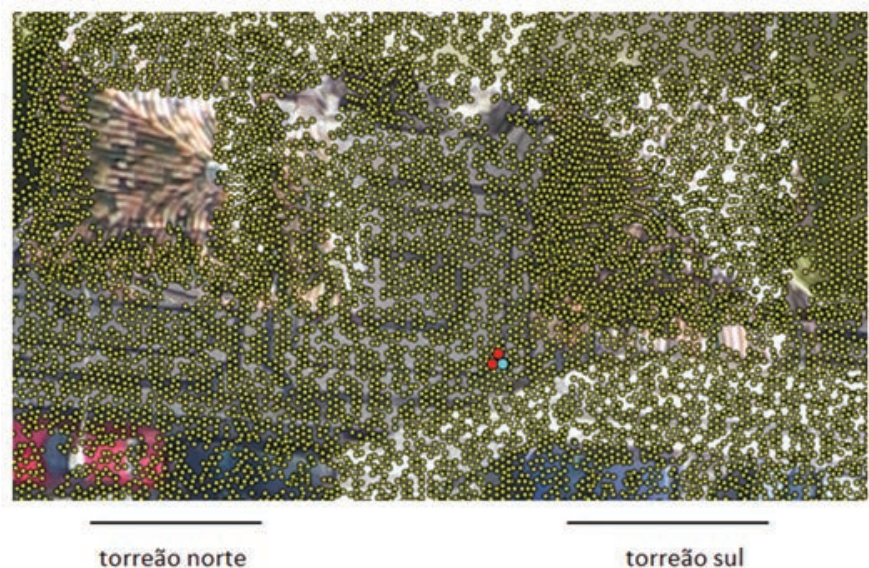

Figura 9 - Os dois telhados dos torreões que delimitam a entrada principal do Parque de Santa Cruz 
Para ter informação altimétrica é necessário criar o MDS, o qual é construído a partir da nuvem de pontos gerada também pelo software T3D, modelo necessário para transformar as fotografias em ortofotos. A nuvem era constituída por 3331688 pontos, distribuídos irregularmente. Cada ponto da nuvem tem coordenadas $\mathrm{M}, \mathrm{P}$ e $\mathrm{H}$ e também a cor da imagem nesse ponto. Esta nuvem foi automaticamente filtrada pelo software, tendo-se obtido uma nova nuvem com um menor número de pontos, 784 381, também distribuídos irregularmente. Desta foi gerado, para visualização, um MDS na forma de uma rede de triângulos (Figura 10).
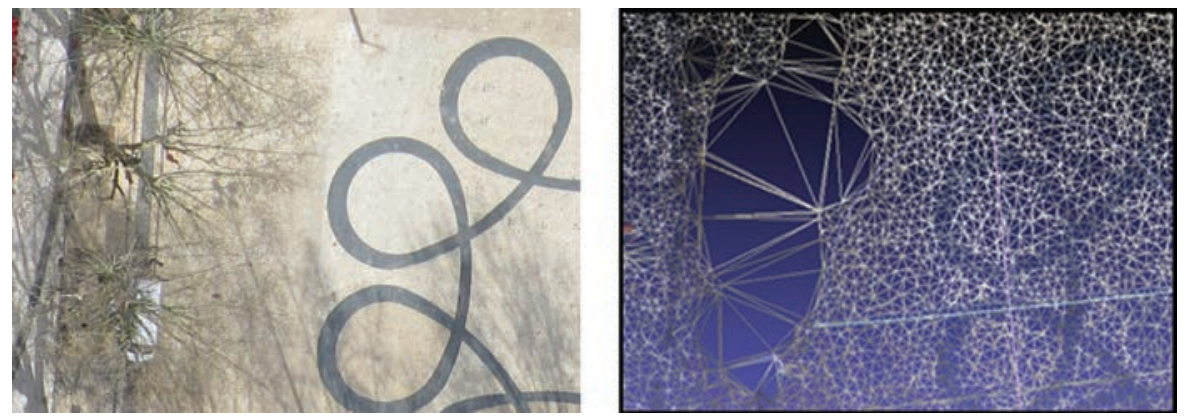

Figura 10 - Praça da República: ortomosaico e rede de triângulos (inclui uma área com triângulos com lados extensos devido à nuvem não ter pontos na zona das árvores)

A visualização da rede de triângulos do MDS permite verificar que não são criadas linhas de quebra do declive ("breaklines") as quais materializam as fronteiras entre zonas que têm inclinações muito diferentes. Por exemplo, os beirais de edifícios são linhas de quebra entre o plano inclinado do telhado e o plano vertical da fachada. A identificação de linhas de quebra, processo que depende fortemente de um operador e que tem uma elevada componente manual, permite a geração de MDS mais robustos, que descrevem melhor a realidade. No MDS criado pelo T3D estas linhas não existem (embora possa haver uma edição manual a fim de as criar), pelo que nas zonas onde há pontos contíguos com altitudes muito diferentes os triângulos apresentam inclinações muito elevadas (Figuras 11 a) e b) ) e nessa zona o ortomosaico apresenta anomalias que não surgem num ortofotomapa verdadeiro, o qual necessariamente inclui, durante a produção, a localização de linhas de quebra. 

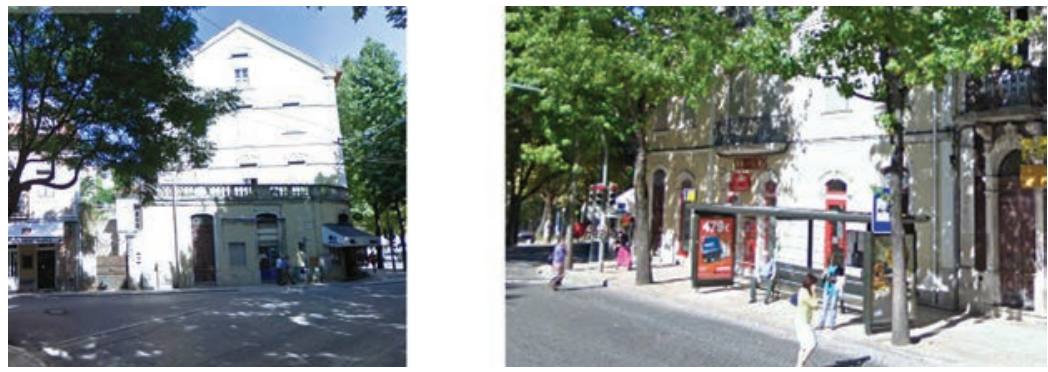

Figura 11 a) - Edifício na esquina da Praça da República com a Rua Tenente Valadim: duas fotografias obtida do Google Maps Street View (visto respetivamente da Rua Tenente Valadim e da Praça da República)
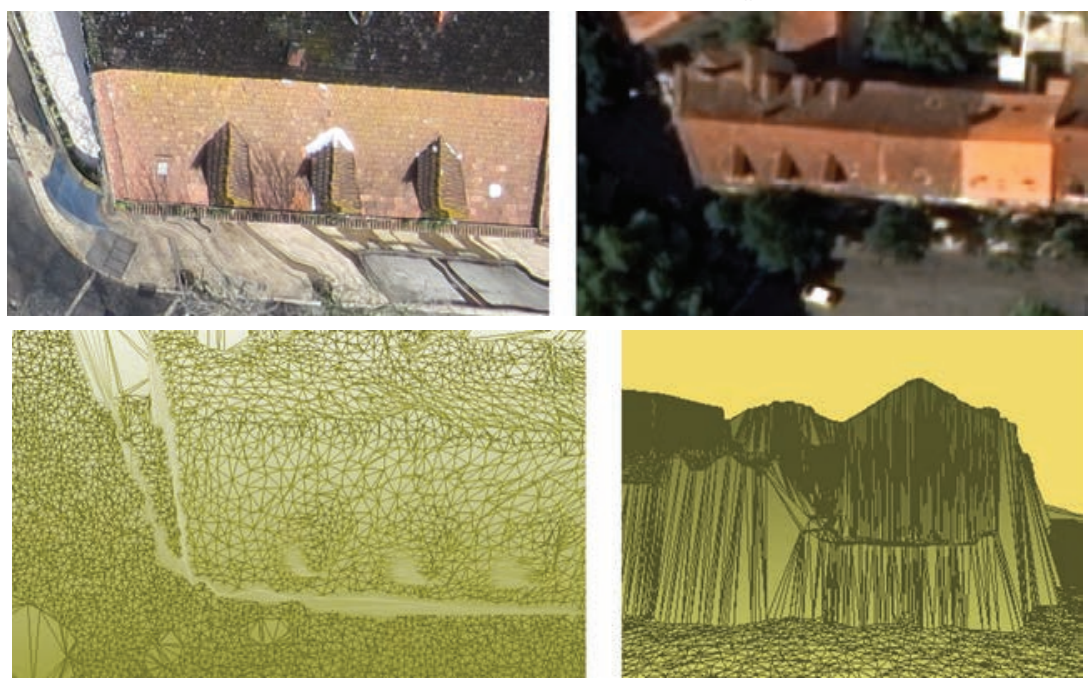

Figura 11 b) - Pormenor do ortomosaico e de um ortofotomapa, rede de triângulos em planta e numa perspetiva

Outros problemas visíveis no ortomosaico, criado por junção de ortofotos, têm origem nas próprias ortofotos. Estes são: i) o duplo mapeamento, repetição de uma secção de uma imagem na imagem e que ocorre nas zonas ocultas por edifícios; e ii) o escorregamento (Habib et al., 2007). Na Figura 12, na área entre as linhas vermelhas (marcadas manualmente pelos autores, o mesmo acontecendo com as azuis) ocorre duplo mapeamento e escorregamento. Esta área corresponde ao passeio que na fotografia original estava encoberto pelo edifício. Na criação do ortomosaico o software escolheu outra ortofoto para aquela área, o que não impede que continuem a ocorrer, embora com menos expressão, os dois problemas referidos. 

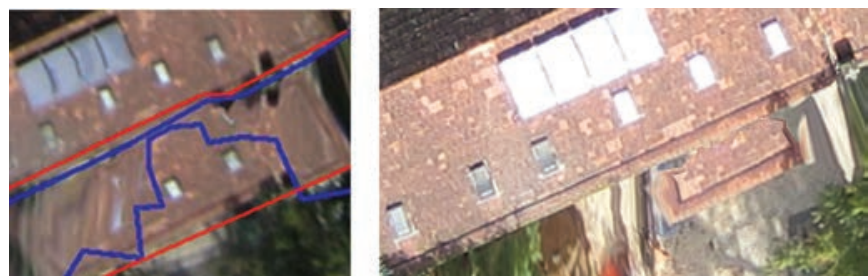

Figura 12 - Ortofoto do telhado de um edifício e área correspondente no ortomosaico. As linhas vermelhas marcam a localização de linhas de quebra; as azuis a fronteira entre zonas de declive muito diferente (ver Figura 15)

O duplo mapeamento ocorre na fase da transformação da fotografia, imagem obtida com uma perspetiva central, numa ortofoto, imagem com uma projeção ortogonal. Considerando o exemplo da Figura 13, onde se representa um edifício implantado num terreno plano (A), a imagem ortogonal da superfície (a que se desejaria obter após a transformação) seria formada por um conjunto de pixéis de igual dimensão com a sequência de cores da superfície (C). Devido ao edifício e à localização do VANT uma área do terreno não fica registada na imagem (B) pois está ocultada pelo edifício. A fachada verde do edifício, que não surgiria numa ortofoto, fica registada na fotografia. Pela posição do VANT, determinada por cálculo, e estabelecido o MDS, o software preenche as zonas que ficaram ocultadas pelo edifício, atribuindo aos pixéis ocultos as cores dos pixéis do topo do edifício. A imagem do topo do edifício fica assim repetida, dando origem ao duplo mapeamento. No preenchimento de "D" na Figura 13 foi considerado que o MDS descreve bem o terreno, ajustando-se perfeitamente a este.

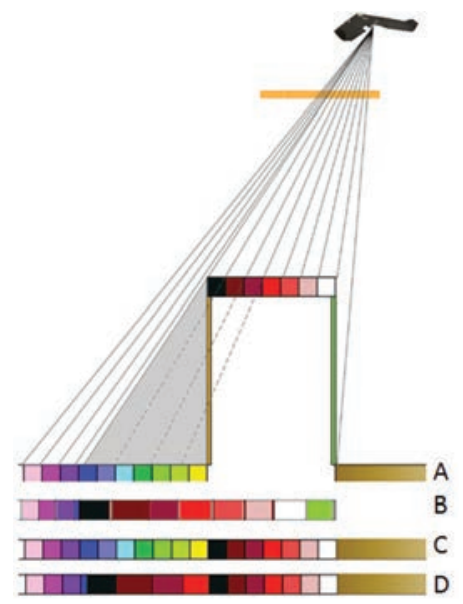

Figura 13 - Edifício sobre um terreno

A: superfície do terreno;

B: imagem obtida no plano da fotografia (ampliada);

C: ortofoto do terreno;

D: imagem do terreno gerada pelo software. 
Quando o MDS é estimado de forma incorreta o preenchimento dos pixéis da ortofoto fica afetado. Na Figura 14 tenta-se ilustrar a forma como o MDS altera a distribuição cromática da imagem. Nesta figura o MDS está representado pela linha verde que une a nuvem de pontos, representados pelos círculos cinzentos e que têm altitudes incorretas (se a altitude destes fosse a correta distribuir-se-iam ao longo de uma linha horizontal). Por exemplo, centrando a atenção no pixel azul da Figura, zona limitada por pontos com um desnível muito acentuado na direção da linha de observação (desnível incorreto como foi afirmado), tem-se que na ortofoto a área a azul ficaria muito extensa, originando a impressão da ocorrência de um escorregamento. Pelo contrário, nas zonas onde o desnível é acentuado mas na direção ortogonal à linha de observação (ocorre por exemplo nas zonas verdes e roxas) há uma compressão da área original. O exemplo apresentado na figura está muito simplificado pois considera-se que os pontos que materializam o terreno (linha horizontal) estão igualmente espaçados sendo que os erros no MDS só resultam de erros nas altitudes dos pontos.

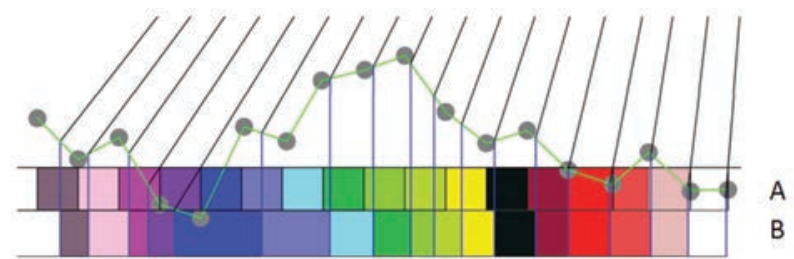

Figura 14 - Superfície original (terreno) é horizontal sendo que o MDS apresenta incorreções em altitude. A: ortofoto correta; B: ortofoto criada a partir do MDS incorreto

$\mathrm{Na}$ realidade os pontos do MDS estão afetados de erros tanto em planimetria como em altimetria pelo que a situação que frequentemente ocorre é a apresentada na Figura 15, dos dois edifícios entre a Praça da República e a Rua Lourenço de Almeida Azevedo. Devido à posição do VANT o passeio está ocultado pelos edifícios. No MDS a empena sul dos edifícios não está vertical mas sim inclinada (como foi referido o software não cria MDS com planos verticais) e a linha que deveria marcar o contacto da empena com o passeio está altamente irregular em resultado do MDS incorporar pontos do topo da paragem de autocarro, do autocarro, do chapéu-de-sol, de toldos, etc. (Figura 15). Nas zonas originalmente 
ocultadas pelos edifícios (zona delimitada a vermelho) foram ocupadas por imagem dos telhados (duplo mapeamento) sendo que se pode notar que dentro da área de duplo mapeamento ocorre uma zona de escorregamento que correspondente à área onde o MDS apresenta uma elevada inclinação (limitada pelas linhas azuis no MDS).

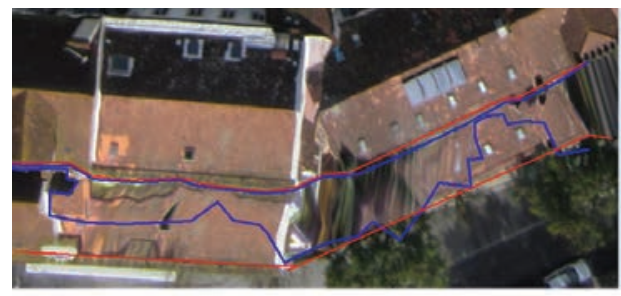

a)

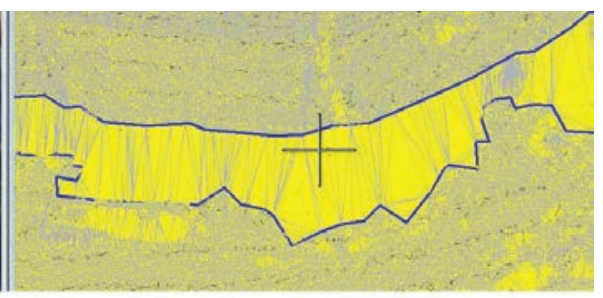

b)

Figura 15 - Uma ortofoto (a) e uma imagem do MDS da zona, desenvolvido em MicroStation (b). As linhas azuis delimitam uma zona com acentuado declive
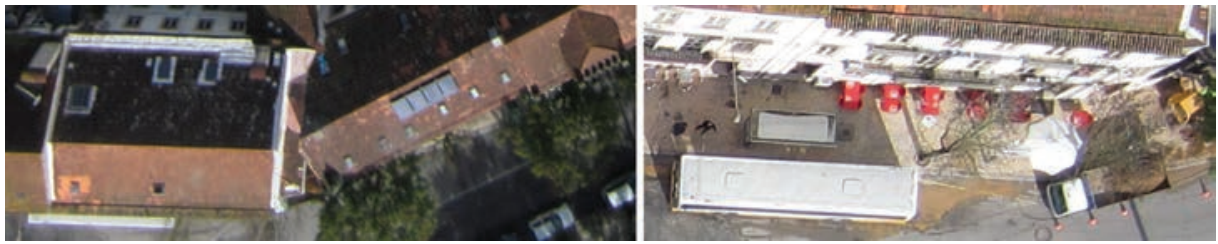

Figura 16 - Fotografia antes da ortorretificação. Elementos situados no passeio

No ortomosaico, obtido por colagem das ortofotos, operação que é realizada automaticamente pelo software, está bem visível que este reúne áreas provenientes de várias ortofotos (Figura 17). Assim, o passeio situado em frente do edifício da esquerda, resulta da união de áreas da ortofoto "A" com a ortofoto "D", as mesmas que foram utilizadas para o telhado (da "A" para a zona esquerda, da "D" para o centro e direita). O utilizador tem possibilidade de, através de uma edição manual, optar por outras ortofotos para diferentes zonas. Na Figura 178 verifica-se que no ortomosaico os edifícios ficariam mais bem representados se o operador tivesse realizado uma edição e escolhido um extrato da ortofoto "E" para preencher a área da direita. 



Figura 17 - Pormenor do ortomosaico de edifícios. As seis ortofotos que cobriram a zona
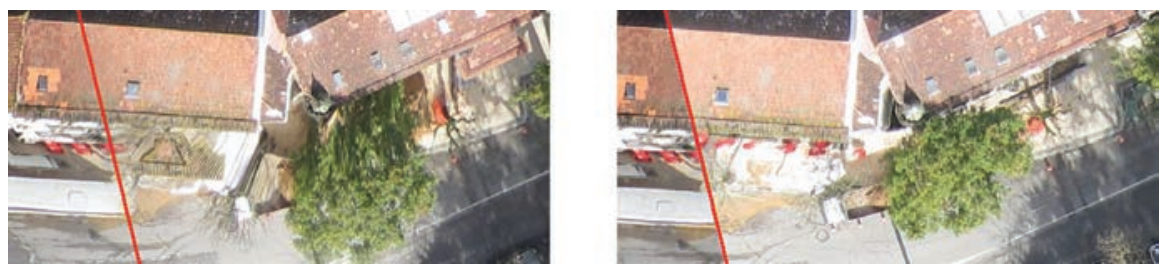

Figura 18 - Pormenor do ortomosaico, criado por junção de áreas das ortofotos A e D, e a hipótese de este resultar da junção de áreas das ortofotos A e E

\section{CONTROLO DA QUALIDADE}

O controlo da qualidade do ortomosaico e do MDS pode ser efetuado por aplicação de testes à exatidão e precisão de dados, testes estes utili- 
zados para avaliar a qualidade posicional (Casaca 1999, Morrison 1990). Os dados são diferenças (desvios) entre coordenadas GPS e coordenadas do ortomosaico/MDS de pontos de controlo (PC) (Figura 19). As coordenadas GPS, estimadas com um erro inferior a $2 \mathrm{~cm}$ em planimetria e $3 \mathrm{~cm}$ em altimetria, são mais exatas que as coordenadas obtidas pelo sistema VANT e por isso são consideradas coordenadas de referência.

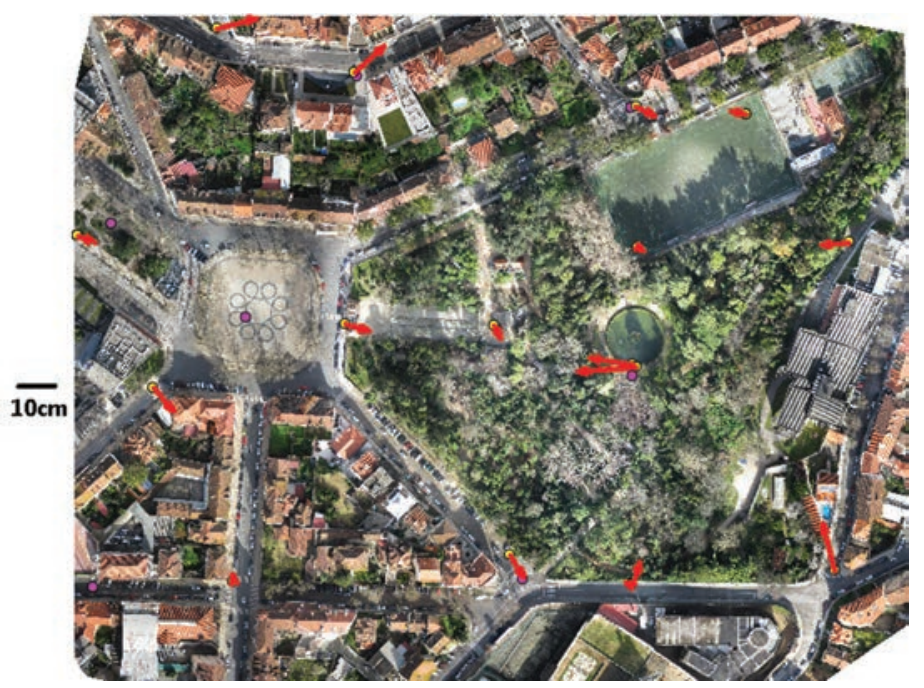

Figura 19 - Ortomosaico com indicação da localização dos pontos fotogramétricos (magenta), dos pontos de controlo (amarelo) e diferenças entre coordenadas planimétricas do ortomosaico e GPS (setas a vermelho)

Quadro I - Diferenças entre coordenadas

\begin{tabular}{cccc|cccc}
\hline Id & $\Delta M(m)$ & $\Delta P(m)$ & $\Delta H(m)$ & Id & $\Delta M(m)$ & $\Delta P(m)$ & $\Delta H(m)$ \\
\hline P01 & 0.079 & 0.004 & -0.053 & $P 10$ & -0.065 & 0.060 & -0.136 \\
P02 & 0.004 & -0.109 & -0.223 & $P 11$ & -0.045 & 0.062 & 0.071 \\
P03 & 0.039 & -0.023 & 0.011 & $P 12$ & -0.075 & -0.053 & -0.076 \\
P04 & -0.011 & 0.021 & 0.053 & $P 13$ & -0.080 & 0.000 & 0.081 \\
P05 & -0.082 & 0.006 & 0.029 & $P 14$ & -0.008 & -0.030 & -0.151 \\
P06 & 0.140 & 0.000 & -0.375 & $P 15$ & -0.127 & -0.032 & -0.050 \\
P07 & -0.032 & -0.077 & 0.015 & $P 16$ & -0.070 & 0.014 & 0.016 \\
P08 & 0.087 & 0.011 & -0.050 & $P 17$ & -0.056 & 0.016 & -0.018 \\
P09 & -0.010 & -0.031 & 0.000 & & & & \\
\hline
\end{tabular}

Dos valores apresentados no Quadro I, calcula-se a média empírica (T em sobrescrito indica que se apresenta o vetor transposto):

$$
\vec{m}^{\top}=\left[\begin{array}{lll}
m_{M} & m_{p} & m_{H}
\end{array}\right]=\left[\begin{array}{lll}
-0.018 m & -0.009 m & -0.050 m
\end{array}\right]
$$


e a matriz de variância (unidade $\mathrm{m} 2$ )

$$
S_{M P H}=\left[\begin{array}{ccc}
S_{M}^{2} & S_{M P} & S_{M H} \\
S_{M P} & S_{P}^{2} & S_{P H} \\
S_{M H} & S_{P H} & S_{H}^{2}
\end{array}\right]=\left[\begin{array}{ccc}
0.0050 & -0.0001 & -0.0043 \\
-0.0001 & 0.0019 & 0.0014 \\
-0.0043 & 0.0014 & 0.0136
\end{array}\right] .
$$

sendo que em Henriques et al. (2014) se apresentam as expressões que permitem realizar o cálculo destes valores.

A aplicação da metodologia habitual, utilizada em muitos países para verificação da aceitação de um produto cartográfico, recorre a uma confrontação entre o valor do erro médio quadrático (EMQ) das diferenças e um valor de referência. Este valor de referência pode ser, por exemplo, o publicado pela Direção Geral do Território, apresentado no Quadro II (DGT, 2014 e referido também em DGT, 2013).

Quadro II - Valores para avaliação da qualidade baseada no EMQ

\begin{tabular}{|l|l|c|c|c|}
\hline & \multicolumn{1}{|c|}{ escalas cartográficas } & $1 / 1000$ & $1 / 2000$ & $1 / 5000$ \\
\hline \multirow{3}{*}{ Orto: planimetria } & EMQ menor que & $0.18 \mathrm{~m}$ & $0.30 \mathrm{~m}$ & $0.75 \mathrm{~m}$ \\
\cline { 2 - 5 } & $\begin{array}{l}\text { pelo menos 90\% dos elementos da amostra } \\
\text { têm de apresentar um desvio menor que }\end{array}$ & $0.27 \mathrm{~m}$ & $0.45 \mathrm{~m}$ & $1.25 \mathrm{~m}$ \\
\hline \multirow{2}{*}{$\begin{array}{l}\text { Nuvem de pontos: } \\
\text { altimetria }\end{array}$} & EMQ menor que & $0.15 \mathrm{~m}$ & $0.25 \mathrm{~m}$ & $0.40 \mathrm{~m}$ \\
\cline { 2 - 5 } & $\begin{array}{l}\text { pelo menos 90\% dos elementos da amostra } \\
\text { têm de apresentar um desvio menor que }\end{array}$ & $0.41 \mathrm{~m}$ & $0.65 \mathrm{~m}$ & $1.65 \mathrm{~m}$ \\
\hline
\end{tabular}

As coordenadas planimétricas dos PC foram obtidas a partir do ortomosaico gerado pelo software T3D. Este foi importado para o software QGIS onde se identificaram os pontos na imagem e se obtiveram as coordenadas planimétricas (M e P). O EMQ de 17 PC foi EMQMP=0.09m sendo que $100 \%$ das diferenças são menores que $0.27 \mathrm{~m}$. A altitude ortométrica $(\mathrm{H})$ foi obtida da nuvem de pontos utilizando o método de interpolação por coordenadas baricêntricas tendo sido obtido $\mathrm{EMQH}=0.13 \mathrm{~m}$ sendo que $100 \%$ das diferenças são menores que $0.41 \mathrm{~m}$.

No entanto o método apresentado só avalia a dispersão das diferenças pelo que se propõe que a avaliação da qualidade posicional deva incidir sobre a exatidão e a precisão das diferenças se baseie em métodos da 
inferência estatística mais completos. A metodologia de teste foi apresentada por Casaca (1999) tendo já sido aplicada numa avaliação a um outro ortomosaico/MDS (Henriques, 2014), obtido em condições de operação muito difíceis.

\subsection{Teste da exatidão}

Seguindo a metodologia descrita nos trabalhos atrás referidos, são calculadas as variáveis v e q, ambas escalares, em que a primeira é calculada com base no vetor de diferenças (1) e na matriz de variância (2), e a segunda representa o quantil de probabilidade 0.95 da distribuição $\mathrm{F}$ de Snedecor com os graus de liberdade f1 e f2: f1 é igual a 1, 2 ou 3 quando se testam, respetivamente, dados unidimensionais (altimétricos por exemplo), planimétricos ou tridimensionais (3D); f2 é igual a n-1, sendo $\mathrm{n}$ o número total de PC. A variável v é calculada por uma das seguintes expressões

$$
v_{M}=n \frac{m_{M}^{2}}{s_{M}^{2}} \therefore ; v_{M P}=\frac{n(n-2)}{2(n-1)} \vec{m}^{\top} S_{M P}{ }^{-1} \vec{m} ; v_{M P H}=\frac{n(n-3)}{3(n-1)} \vec{m}^{\top} S_{M P H}{ }^{-1} \vec{m}
$$

O símbolo é aplicado para indicar que as expressões para as restantes variáveis (neste caso $v_{P}$ e $v_{H}$ ) são equivalentes à expressão apresentada. As variáveis incluídas nas expressões apresentadas são obtidas de (1) e (2).

No teste, a variável v é comparada com as regiões de aceitação (RA) e região crítica $(\mathrm{RC})$, sendo que se aceita a hipótese da amostra ser exata quando a variável de teste está incluída na região de aceitação:

$$
R A=[0, q], R C=] q,+\infty[
$$

Quadro III - Teste da exatidão

\begin{tabular}{c|c|c}
\hline $\mathrm{v}_{M}=1.11$ (RA) & $\mathrm{v}_{M P}=0.87$ (RA) & $\mathrm{v}_{M P H}=2.45$ (RA) \\
$\mathrm{v}_{\mathrm{P}}=0.71$ (RA) & $\mathrm{q}=3.68$ & $\mathrm{q}=3.34$ \\
$\mathrm{v}_{\mathrm{H}}=3.11$ (RA) & & \\
$\mathrm{q}=4.49$ & & \\
\hline
\end{tabular}

Verificando os valores apresentados no Quadro III aceita-se a exatidão do ortomosaico e do MDS. 


\subsection{Teste da dispersão}

Neste teste avalia-se se o ortomosaico e o MDS estão incluídos numa classe de precisão pré-estabelecida, caracterizada por uma tolerância, com um nível de qualidade aceitável, por exemplo 0.95. A variância $\sigma_{\mathrm{J}}^{2}$ de cada classe "J" de precisão é determinada por

$$
\sigma_{\mathrm{f}}^{2}=\frac{\left(\mathrm{t}_{\mathrm{j}}\right)^{2}}{\mathrm{q}}
$$

q é o quantil de probabilidade 0.95 da distribuição Qui-quadrado com $\mathrm{f}$ graus de liberdade. Neste teste cada componente ( $\mathrm{M}$, ou $\mathrm{P}$ ou $\mathrm{H}$ ) pode ser testada individualmente, e neste caso $\mathrm{f}=1$, ou o teste pode incidir sobre coordenadas $2 \mathrm{D}$ ou $3 \mathrm{D}$ ( $\operatorname{com} \mathrm{f}=2$ ou $\mathrm{f}=3$, respetivamente). No Quadro IV apresentam-se os valores de variância para várias classes de tolerância.

Quadro IV - Valores da variância para classes de tolerância

\begin{tabular}{|c|c|c|c|c|}
\hline & $t_{1}=0.05 m$ & $\mathrm{t}_{\| 1}=0.10 \mathrm{~m}$ & $\mathrm{t}_{\mathrm{III}}=0.15 \mathrm{~m}$ & $\mathrm{t}_{\mathrm{IV}}=0.20 \mathrm{~m}$ \\
\hline $1 D: q=3.84$ & $\sigma_{1}^{2}=0.00065 \mathrm{~m}^{2}$ & $\sigma_{\|}^{2}=0.00260 \mathrm{~m}^{2}$ & $\sigma_{\| I}^{2}=0.00586 \mathrm{~m}^{2}$ & $\sigma_{\mathrm{IV}}^{2}=0.010417 \mathrm{~m}^{2}$ \\
\hline $2 D: q=5.99$ & $\sigma_{1}^{2}=0.00043 m^{2}$ & $\sigma_{\|}^{2}=0.00167 \mathrm{~m}^{2}$ & $\sigma_{I I I}^{2}=0.00376 \mathrm{~m}^{2}$ & $\sigma_{\mathrm{IV}}^{2}=0.00668 \mathrm{~m}^{2}$ \\
\hline
\end{tabular}

Seguindo a metodologia descrita nos trabalhos atrás referidos, são calculadas as variáveis u e q, ambas escalares. A primeira é calculada com base na matriz (2) e na variância associada a cada classe de tolerância:

$$
\left(\mathrm{u}_{M}\right)_{J}=(\mathrm{n}-1) \frac{s_{M}^{2}}{\sigma_{M, 1}^{2}} \therefore
$$

A segunda variável representa o quantil de probabilidade 0.95 da distribuição Qui-quadrado com (n-1) graus de liberdade. Analogamente ao teste anterior são também consideradas a região de aceitação e a região de crítica. No Quadro V, onde são apresentados os resultados dos testes, RA ou RC indicam se, de acordo com o teste, o ortomosaico ou o MDS podem ser utilizados em aplicações que exijam a tolerância indicada. Por exemplo, se for utilizada unicamente a informação planimétrica, o ortomosaico produzido poderia ser utilizado em trabalhos cuja tolerância fosse $0.15 \mathrm{~m}$. 
Quadro V - Testando a precisão de cada componente

\begin{tabular}{|c|c|c|c|}
\hline $\mathrm{t}_{\mathrm{l}}=0.05 \mathrm{~m}$ & $\mathrm{t}_{1 \mid}=0.10 \mathrm{~m}$ & $\mathrm{t}_{\mathrm{III}}=0.15 \mathrm{~m}$ & $\mathrm{t}_{\mathrm{iv}}=0.20 \mathrm{~m}$ \\
\hline$u_{M}=122.88(R C)$ & $\mathrm{u}_{\mathrm{M}}=30.72(\mathrm{RC})$ & $\mathrm{u}_{\mathrm{M}}=13.65(\mathrm{RA})$ & $\mathrm{u}_{\mathrm{M}}=7.68(\mathrm{RA})$ \\
\hline$u_{p}=46.69(R C)$ & $u_{p}=11.67(R A)$ & $u_{p}=5.19(R A)$ & $u_{p}=2.92(R A)$ \\
\hline $\mathrm{u}_{\mathrm{H}}=334.23(\mathrm{RC})$ & $\mathrm{u}_{\mathrm{H}}=83.56(\mathrm{RC})$ & $\mathrm{u}_{\mathrm{H}}=37.14(\mathrm{RC})$ & $u_{H}=20.89$ (RA) \\
\hline$q=26.30$ & $q=26.30$ & $q=26.30$ & $q=26.30$ \\
\hline
\end{tabular}

De acordo com Casaca (1999) a tolerância planimétrica deve ser definida em função de um valor constante no "papel", por exemplo $0.5 \mathrm{~mm}$. A tolerância altimétrica deve ser definida em função da equidistância gráfica, por exemplo, um quinto desta equidistância. Nestas condições, uma planta topográfica à escala 1:1 000, deve apresentar:

i) Erros planimétricos inferiores a $0.5 \mathrm{~m}$, à escala.

ii) Erros altimétricos inferiores a um quinto da equidistância gráfica convencional $(1 \mathrm{~mm})$, ou seja $0.2 \mathrm{~m}$, à escala.

Em face destes valores e comparando com os apresentados no Quadro I verifica-se que as componentes $\mathrm{M}, \mathrm{P}$ e $\mathrm{H}$ se incluem nestas classes de tolerância pelo que o ortomosaico e MDS gerados a partir das fotografias obtidas pelo VANT seriam aceites.

Para fazer uma análise mais completa, não baseada numa análise das coordenadas como unidades independentes mas sim nas coordenadas planimétricas (2D), Casaca adapta a metodologia apresentada por Maling (1989), o qual usa uma comparação das elipses com igual densidade de probabilidade da distribuição das diferenças com os círculos de tolerância planimétrica.

A primeira fase implica calcular os valores próprios máximo e mínimo da inversa da matriz de variância, o que pode ser realizado utilizando as seguintes expressões (Morrison, 1990).

$L_{\max }=\frac{S_{M}^{2}+S_{P}^{2}+\sqrt{\left(S_{M}^{2}-S_{P}^{2}\right)^{2}+4\left(S_{M P}\right)^{2}}}{2\left[S_{M}^{2} S_{P}^{2}-\left(S_{M P}\right)^{2}\right]}$

$$
L_{\min }=\frac{S_{M}^{2}+S_{P}^{2}-\sqrt{\left(S_{M}^{2}-S_{P}^{2}\right)^{2}+4\left(S_{M P}\right)^{2}}}{2\left[S_{M}^{2} S_{P}^{2}-\left(S_{M P}\right)^{2}\right]}
$$


A segunda fase envolve calcular valores para as estimativas de $\mathrm{L}_{\min }$, $\lambda_{*}$ e $\lambda_{0}$ sendo estas duas últimas calculadas por

$$
\lambda_{*}=L_{\min }\left[1-\frac{L_{\max }}{(n-1)\left(L_{\min }-L_{\max }\right)}\right]
$$

O teste envolve confrontar o inverso dos valores assumidos pelas três estimativas com o valor da variância, apresentado no Quadro 4 (valores relativos a 2D), associado às tolerâncias pré-estabelecidas. Substituindo os valores da matriz de variância nas expressões apresentadas obtém-se

$$
\begin{gathered}
\hat{L}_{M A X}=527.21 \mathrm{~m}^{-2} ; \hat{L}_{\mathrm{MIN}}=199.87 \mathrm{~m}^{-2} ; 1 / \hat{L}_{\mathrm{MIN}}=0.0051 \mathrm{~m}^{2}, \\
\hat{\lambda}_{*}=219.99 \mathrm{~m}^{-2} ; 1 / \hat{\lambda}_{*}=0.00460 \mathrm{~m}^{2}, \\
\hat{\lambda}_{0}=222.33 \mathrm{~m}^{-2} ; 1 / \hat{\lambda}_{0}=0.0045 \mathrm{~m}^{2} .
\end{gathered}
$$

Verificando-se que

$$
\sigma_{\mathrm{III}}^{2}<1 / \hat{L}_{\mathrm{Min},} 1 / \hat{\lambda}^{*}, 1 / \hat{\lambda}_{0}<\sigma_{\mathrm{IV}}^{2}
$$

aceita-se que o ortomosaico fique incluído na classe IV de tolerância.

\section{CURVAS DE NÍVEL}

Como foi referido, a componente altimétrica dos dados permite a geração de um MDS. Este diferencia-se do modelo digital do terreno pois neste último são retirados todos os objetos (edifícios, veículos, árvores, etc.) que se encontram sobre a superfície terrestre.

Todo o processamento realizado no presente trabalho foi totalmente automático. Como se mostrou, por razões relacionadas com a distribuição espacial dos pontos, não é possível existirem planos verticais num MDS pelo que a não marcação de linhas de quebra dos edifícios leva a que o MDS não represente bem a superfície. Por exemplo, na Figura 10 vê-se que a fachada principal de um dos edifícios da Praça da República está 
representada por superfícies inclinadas quando na realidade a fachada é uma superfície vertical. E o facto de não serem marcadas linhas de quebra e não serem "retirados" os objetos que se encontram sobre o terreno, leva a que o MDS apresente o que parecem ser anomalias (algumas estão visíveis na Figura 20: por exemplo, no Parque existem pontos nas copas das árvores).

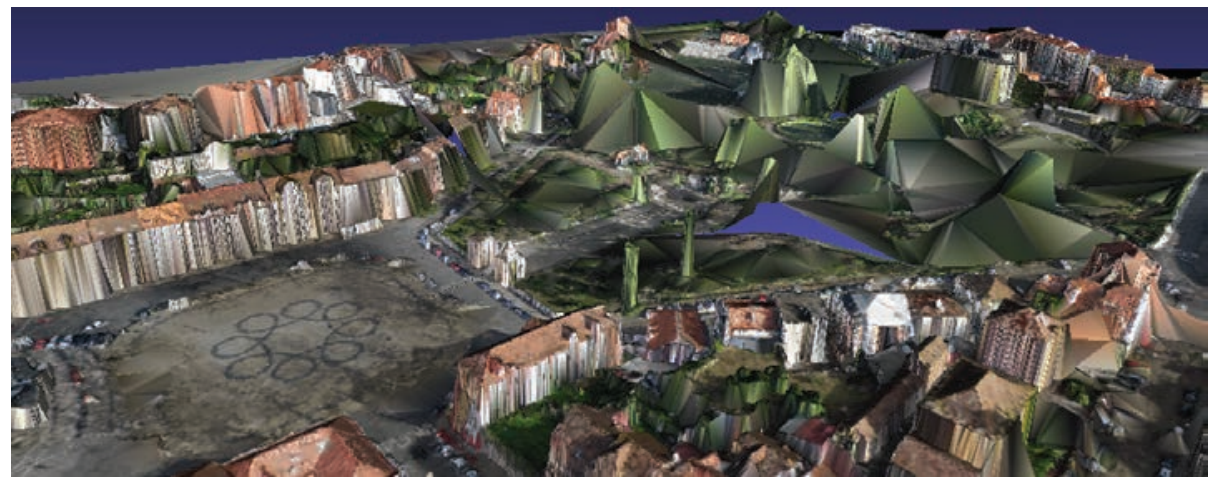

Praça da República

Parque de Santa Cruz

Figura 20 - Representação tridimensional do MDS (Praça da República e Parque)

Estas “anomalias” repercutem-se nos produtos cartográficos gerados a partir do MDS, como é o caso das curvas de nível. Estas refletem necessariamente todos os objetos que existem sobre o terreno pelo que a primeira visão da distribuição das curvas causa muita estranheza, principalmente quando as curvas são comparadas com as curvas de nível representadas numa planta tradicional, curvas estas relativas ao Modelo Digital do Terreno (diferente do MDS pois este último inclui todos os "objetos" situados sobre o terreno: edifícios, automóveis, bancos, etc.). $\mathrm{Na}$ Figura 21 apresentam-se as curvas de nível geradas automaticamente a partir da nuvem de pontos utilizando o programa QGIS tendo sido criado, como passo intermédio obrigatório, uma grelha regular em que a cada célula é atribuída uma altitude. Dado o grande volume de dados (mais de três milhões de pontos) a grelha foi criada pelo método de interpolação "TIN” (Triangular Irregular Network). 




Figura 21 - Curvas de nível, equidistantes $1 \mathrm{~m}$, sobre o ortomosaico

\section{CONCLUSÕeS}

Os produtos cartográficos desenvolvidos a partir de um levantamento fotogramétrico, como as fotografias obtidas por uma câmara digital instalada num VANT, de uma área urbana pouco extensa revelaram-se compatíveis com as exigências da escala 1/1000. Tal foi comprovado através da análise das diferenças entre coordenadas fotogramétricas (ortomosaico e MDS) e coordenadas GPS, análise esta realizada quer aplicando um método simples, baseado numa confrontação do valor do EMQ com um valor de referência, quer num teste mais complexo, que recorre a métodos de inferência estatística para avaliar a exatidão e precisão.

Os produtos cartográficos produzidos, gerados de forma totalmente automática, mostram, no entanto, algumas limitações, sendo que as mais visíveis (duplo mapeamento e escorregamento ${ }^{2}$ ) poderão ser eliminadas se houver, durante a fase de produção, a edição manual do MDS de forma a definir a localização das linhas de quebra (edição que originará, consequentemente, um aumento do custo de produção). O próprio voo

\footnotetext{
2 Parâmetros diferentes dos utilizados no processamentos ou outros softwares poderão resultar no não aparecimento do duplo mapeamento e/ou do escorregamento.
} 
do VANT, realizado a muito baixa altura e por uma aeronave muito leve, origina a captação de fotografias com inclinações elevadas, situação que leva a que seja necessário utilizar software capaz de processar imagens com esta geometria de aquisição desfavorável. Considerando a utilização de uma determinada câmara, um voo realizado a baixa altura leva ainda a que os problemas originados pela ocultação afetem áreas maiores do que em voos realizados a alturas mais elevadas.

Em comparação com os ortofotomapas obtidos a partir de aviões e câmaras utilizadas na fotogrametria clássica, os produtos gerados utilizando um VANT apresentam uma menor qualidade. No entanto o preço (que é na verdade muito baixo quando comparado com os custos da produção fotogramétrica por métodos convencionais) e a facilidade com que pode ser obtido (o manuseamento da aeronave e, posteriormente, o processamento das imagens são operações muito simples e bastante intuitivas para quem tiver tido contacto com os métodos clássicos de levantamento fotogramétrico) são a mais-valia que faz com que a sua utilização deva ser equacionada em aplicações sobre áreas pouco extensas, mesmo quando exista uma exigência elevada da qualidade do produto final.

\section{BIBLIOGRAFIA}

CASACA, João (1999) - A avaliação da qualidade posicional de cartografia topográfica em escalas grandes. Atas da II Conferência Nacional de Cartografia e Geodesia.

DIREÇÃO GERAL DO TERRITÓRIO (2013) - Cartografia e Ortofotocartografia à Escala 1:2 000. Normas técnicas de produção e reprodução. Direção Geral do Território. Consultado em junho de 2014. Disponível no endereço www.dgterritorio.pt/ficheiros/dgt/especificacoes_tecnicas_2k_com_catalogo_20130601_pdf

DIREÇÃO GERAL DO TERRITÓRIO (2014) - Exatidão e precisão posicionais para a cartografia em escalas grandes. Direção Geral do Território. Consultado em fevereiro de 2014. Disponível no endereço www.dgterritorio.pt/ficheiros/ dgt/internet_precisoes_para_1k_2k_5k_10kjun2013_pdf

HABIB, Ayman, KIM, Eui-Myoung Kim, \& KIM, Chang-Jae (2007) - New methodologies for true orthophoto generation. Photogrammetric Engineering \& Remote Sensing Vol. 73, No. 1, pp. 025-036. 
HENRIQUES, Maria João et al. (2014) - Assessing the quality of an UAV-based orthomosaic and surface model of a breakwater. FIG Congress 2014. Disponível no endereço http://www.fig.net/pub/fig2014/techprog.htm.

MORRISON,D. (1990). Multivariate statistical methods. McGraw-Hill.

MALING, Derek Hylton (1989) - Measurement from maps: principles and methods of cartometry. Pergamon Press.

STRECHA, Christoph (2011) - Automated Photogrammetric Techniques on Ultralight UAV Imagery. 53rd Photogrammetric Week, pag. 289-294.

VALLET, Julien et al. (2011) - Photogrammetric performance of an ultra light weight swinglet "UAV", Int. Arch. Photogramm. Remote Sens. Spatial Inf. Sci., XXXVIII-1/C22, 253-258, doi:10.5194/isprsarchives-XXXVIII-1-C22-253-2011. 
Série Documentos

Imprensa da Universidade de Coimbra

Coimbra University Press

2015

- U M

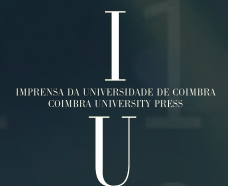

